hep-th/0105227

EFI-01-15

UCLA $/ 01 /$ TEP $/ 9$

\title{
Loop Corrected Tachyon Condensation
}

\author{
Ben Craps $^{1}$, Per Kraus ${ }^{2}$ and Finn Larsen ${ }^{1}$ * \\ ${ }^{1}$ Enrico Fermi Institute, University of Chicago, 5640 S. Ellis Av., Chicago, IL-60637 \\ ${ }^{2}$ Department of Physics, University of California, Los Angeles, CA 90095
}

\begin{abstract}
We study loop corrections in boundary string field theory (BSFT). After commenting on problems with quantizing the tree level BSFT as an ordinary field theory, we discuss the tree level coupling to closed strings and define the loop corrections via factorization in the closed string channel. This description is weakly coupled in the vicinity of the closed string vacuum. Our proposal for the one-loop effective action differs in general from computing the annulus or cylinder partition functions. We also compute the decay rates and the loop corrections to the tensions of unstable branes in perturbative string theory.
\end{abstract}

May, 2001

* Address after July 1, 2001: Michigan Center for Theoretical Physics, University of Michigan, Ann Arbor, MI-48109. 


\section{Introduction}

Tachyon condensation provides a natural arena for the study of off-shell string theory [1,22,3]. Important intuition about classical string field theory has been developed in this concrete setting. However, some of the most interesting issues raised by the study of open string tachyon condensation involve the nature of the quantum theory:

(1) What is the significance of the closed strings? They appear as poles in open string loop diagrams and they are the only viable excitations remaining after the open strings condense into their vacuum state, but there is no satisfactory understanding of closed strings in classical open string field theory. See [4, [5, 6, ], 8,9,9,10] for various points of view.

(2) Is the open string vacuum strongly coupled? The spacetime action suggests a large effective coupling $\sim 1 / V(T)$ near the vacuum at $V(T)=0$ [11, 12, 13, 14, 15], but the world-sheet expansion indicates weak coupling, because holes are weighted by $\sim V(T)$ [16, 17].

(3) Are unstable branes meaningful at finite coupling? Duality relations between distinct unstable theories have been proposed and could be extremely important (e.g. [18, 19,20]), but to make them precise one must define such theories even at large coupling where quantum corrections dominate.

Questions like these force us to confront subtleties of string field theory that are absent in classical computations.

The most convenient framework for our considerations is boundary string field theory (BSFT) [21,22, 23, 24, 25, 17]. BSFT has so far provided a good understanding of tachyon condensation at the classical level, based on extending the conformal sigma model approach to field theories that break conformal invariance on the boundary of a world-sheet with disk topology. The question we shall address in this paper is how to include quantum corrections in BSFT.

To develop intuition about what corrections can be expected, we first consider the one-loop correction to the tension of an unstable D-brane without any world-volume fields excited. This system can be studied in standard perturbative string theory, so that we can temporarily avoid the subtleties associated with going off-shell. The relevant annulus amplitude naïvely diverges due to the exchange of light closed string states as well as the open-string tachyon running in the loop. However, these divergences can be removed by the Fischler-Susskind mechanism [26,27] and analytical continuation [28], respectively. The 
analytic continuation generates an imaginary part, which is interpreted as the decay width of the brane. An amusing qualitative remark is that the decay of the brane is spatially inhomogeneous, in contrast to the "roll down the hill" usually implied in discussions of tachyon condensation. These results are in fact quite familiar in other contexts [29, 30]; we review them in section 2 because only some of them have previously been adapted to the discussion of tachyon condensation in string theory [28].

One perspective on open string field theory is that it is the generalization of standard quantum field theory to a situation with infinitely many fields, one for each open string mode. A natural starting point for quantization is thus a classical action depending on all the open string modes. However, when studying quantum corrections in this way, one has to make sure that one really starts from the full classical action with all fields included, as opposed to an effective action with some of the fields having been integrated out. In section 3, we discuss this approach to quantum string field theory in the context of BSFT. We will be left with unresolved issues that render it unclear (to us) whether this program can be carried out in BSFT. If it could, it would seem to lead to a description that is strongly coupled around the closed string vacuum.

We therefore seek an alternative determination of loop corrections to classical BSFT. Since the classical BSFT action is essentially given by the partition function on the disk, one might expect that the first loop correction corresponds to the partition function on a world-sheet of cylinder topology. However, because the boundary interactions break conformal invariance this result would depend on the choice of Weyl factor. In section 4 we discuss problems with apparently natural choices of world-sheets [31,32]. We are led to the physical condition that open strings must couple consistently to closed strings even off-shell. This condition can be satisfied by starting with the tree-level couplings to closed strings and defining loop amplitudes by demanding factorization in the closed string channel. A feature of this definition is that it leads to a description that is weakly coupled around the closed string vacuum.

The paper is organized as follows. In section 2 we perform the computation of the on-shell loop amplitude for an unstable brane and clarify its physical meaning. Then, in section 3, we review classical BSFT and explore whether a classical action can be obtained that would be a good starting point for quantization. Finally, in section 4 we advance our proposal for one-loop BSFT, based on the requirement of off-shell factorization in the closed string channel. 


\section{One Loop Corrections to the D-brane Tension}

In this section we derive the one-loop correction to the tachyon potential at the unstable maximum with vanishing tachyon $T=0$; in other words, we compute the one-loop correction to the D-brane tension. This is an on-shell question which can be answered in detail without considering the subtleties arising when conformal invariance is broken by the tachyon background.

\subsection{Introductory Remarks}

D-branes are solutions of classical string theory with tensions proportional to $1 / g_{s}$. We want to compute the leading quantum correction to this result. Of course, for BPS D-branes all corrections to the tension will vanish, so to have a nontrivial problem we should consider a non-BPS D-brane. A distinction should be made between those nonBPS D-branes which are classically stable versus those which are classically unstable due to an open string tachyon. Since our ultimate goal is to connect with issues concerning tachyon condensation we focus on unstable D-branes, in particular those of the bosonic string. However, our considerations could also be applied to stable D-branes (for another analysis see [33]).

So we will analyze the question: what is the leading quantum correction to the tension of a bosonic D-brane? Since these D-branes are unstable one does not expect them to exist as energy eigenstates in the full quantum theory. As is familiar, when one tries to compute the energy of such an object one finds a complex number, with the imaginary part being related to the object's decay rate. This will also turn out to be the case here.

The order $\left(g_{s}\right)^{0}$ correction to the D-brane tension will get contributions from various sources. Before going into concrete computations, it is useful to discuss the structure of the expected corrections. Consider a $p$-dimensional object coupled to $D$-dimensional gravity as follows (this should be thought of as part of a low energy effective action of string theory with a D $p$-brane source):

$$
S=\frac{1}{g_{s}^{2}} \int d^{D} x \sqrt{G} R+\tau \int_{p+1} d^{p+1} \xi \sqrt{\tilde{G}} L_{\text {brane }},
$$

where $\tilde{G}$ is the pull-back of the spacetime metric to the D-brane world-volume. First work in the limit of small $g_{s}$ and take the source to have tension $\tau=\tau^{(-1)} / g_{s}$ in this limit $\left(\tau^{(-1)}\right.$ is a constant). The backreaction of the source on the metric can be ignored in the $g_{s} \rightarrow 0$ limit, so we can take $G_{\mu \nu}=\eta_{\mu \nu}$. Now when $g_{s}$ is non-zero, there will be 
quantum corrections to the parameter $\tau$ in (2.1) (corresponding to loops in the D-brane world-volume theory),

$$
\tau=\frac{\tau^{(-1)}}{g_{s}}+\tau^{(0)}+g_{s} \tau^{(1)}+\ldots
$$

and there will be backreaction on the metric,

$$
G_{\mu \nu}=\eta_{\mu \nu}+g_{s} h_{\mu \nu}^{(1)}+\left(g_{s}\right)^{2} h_{\mu \nu}^{(2)}+\ldots
$$

The total tension of the brane, up to order $\left(g_{s}\right)^{0}$, will schematically look like

$$
\frac{\tau^{(-1)}}{g_{s}}+\tau^{(0)}+\tau^{(-1)} h^{(1)}+\left(h^{(1)}\right)^{2} .
$$

The last term in (2.4) corresponds to the energy in the gravitational field excited by the brane. In most of this section we shall concentrate on the second and third term, which come from the first loop correction to the matter energy momentum tensor.

In string theory, one considers a sigma model with background metric (2.3) (and analogously with background values for the dilaton, which we should also include in this discussion). Working perturbatively in $g_{s}$, one can also work with a sigma model in Minkowski background and account for the gravitational background by inserting "Fischler-Susskind" (FS) graviton vertex operators in amplitudes. The classical tension of a D-brane, corresponding to the first term in (2.4), stems from the disk without FS vertex operators. The second term in (2.4) comes from the cylinder (without FS vertex operators), whereas the third term arises from the disk with an order $g_{s}$ FS vertex operator. The fourth term corresponds to the sphere with two order $g_{s}$ FS vertex operators.

In the case of a space-filling D-brane, it turns out that the second term in (2.4) has a divergence due to the exchange of light closed string modes. This divergence is cancelled by the contribution of the disk with an FS vertex operator. Historically, this is how it was discovered that including world-sheets with boundaries leads to modifications of the classical conformal invariance conditions [27]. A similar divergence cancellation mechanism was earlier discovered by Fischler and Susskind [26]. From a modern perspective, the shift in the sigma model metric is nothing but the backreaction of a D-brane on the space-time geometry.

\subsection{The Cylinder Amplitude}

For definiteness we consider a spacefilling D25-brane in bosonic string theory. The leading order tension is equal to the disk partition function, with the result $\tau \sim\left(\alpha^{\prime}\right)^{-13} / g_{s}$. 
The vacuum amplitude on the cylinder is given by the well known expression

$$
Z_{\mathrm{cyl}}=i V_{26} \int_{0}^{\infty} \frac{d t}{2 t}\left(8 \pi^{2} \alpha^{\prime} t\right)^{-13} \eta(i t)^{-24}=\frac{i V_{26}}{2 \pi\left(8 \pi^{2} \alpha^{\prime}\right)^{13}} \int_{0}^{\infty} d s \eta(i s / \pi)^{-24}
$$

where

$$
\eta(i x)^{-24}=\exp (2 \pi x) \prod_{n=1}^{\infty}[1-\exp (-2 \pi n x)]^{-24}=\exp (2 \pi x)+24+O(\exp (-2 \pi x))
$$

Recall that large $t$ and large $s$ correspond to the limits of short and long cylinders respectively. The cylinder amplitude diverges in both of these limits; the large $t$ divergence is due to the open string tachyon, while the large $s$ divergence is due to the closed string tachyon, dilaton and graviton. These two divergences are rendered finite by different mechanisms, which we now review.

We begin with the large $s$ divergence. Imposing a cutoff $s=\ln \Lambda_{s}$ on the upper range of the $s$ integration, the divergent contributions are

$$
Z_{\mathrm{cyl}}^{\mathrm{div}}=\frac{i V_{26}}{2 \pi\left(8 \pi^{2} \alpha^{\prime}\right)^{13}}\left(\frac{1}{2} \Lambda_{s}^{2}+24 \ln \Lambda_{s}\right)
$$

The divergences arise because the D-brane acts as a zero momentum source for the closed string tachyon, dilaton, and graviton. The self energy correction then involves evaluating propagators for these fields at zero momentum, which yields a divergent result in the proper time representation:

$$
\left(\frac{1}{k^{2}+m^{2}}\right)_{k^{2}=0} \longrightarrow \frac{\alpha^{\prime}}{2} \int_{0}^{\ln \Lambda_{s}} d s e^{-\frac{1}{2} \alpha^{\prime} m^{2} s} \sim\left\{\begin{array}{cc}
\Lambda_{s}^{2}, & \alpha^{\prime} m^{2}=-4 \\
\ln \Lambda_{s}, & \alpha^{\prime} m^{2}=0
\end{array}\right.
$$

Since we are ultimately more interested in the superstring, we will focus on the log divergence due to massless closed string exchange. In field theory one finds the same divergences when one tries to expand around a constant field configuration that is not a solution to the equations of motion, and the resolution is simply that one should shift the field to an extremum of the potential. The string theory analogue is the Fischler-Susskind mechanism, which instructs one to cancel the divergences by evaluating amplitudes in a nontrivial closed string background. Thus on the disk one should add a vertex operator proportional to $h_{\mu \nu} \partial X^{\mu} \bar{\partial} X^{\nu}$. Here $h_{\mu \nu} \sim g_{s} \ln \Lambda_{s} \eta_{\mu \nu}$ is the background produced by a constant source for the dilaton and graviton, and is logarithmically divergent as above due 
to the zero momentum singularity of the propagator. If one equates $\Lambda_{s}$ with the worldsheet cutoff then one can think of the Fischler-Susskind mechanism as yielding a loop correction to the world-sheet beta functions, or equivalently, as adding source terms to the dilaton and graviton equations of motion. Similarly, the quadratic divergence is removed by shifting the closed string tachyon.

So the full amplitude at order $\left(g_{s}\right)^{0}$ is obtained by adding together the cylinder, the disk with the insertion of one closed string vertex operator, and the sphere with the insertion of two closed string vertex operators. The sum is what one would measure as the correction to the D-brane tension (see (2.4)). In fact, for a spacefilling D-brane the situation is more complicated. The actual closed string background would involve a rolling dilaton that becomes large somewhere in spacetime, thus invalidating our perturbation theory built around flat spacetime with constant weak string coupling. Symptomatic of this is the fact that our sphere contribution is ill-defined due to the divergent coefficient of the closed string vertex operator. Keeping this in mind, we will in the following just focus on the open string contribution; that is, the cylinder contribution with divergences cancelled against the disk as above. It is this quantity — which essentially gives the zero point energy of open strings on the D-brane - that is the closest analogue of the standard field theory version of the one loop effective potential. In any event, any realistic computation of, say, the tension of a non-spacefilling D-brane, will involve computing the analogue of this quantity.

\subsection{Interpretation of the Open String Tachyon Divergence}

At this stage our cylinder amplitude has been rendered finite in the large $s$ limit and reads

$$
\begin{aligned}
\tilde{Z}_{\mathrm{cyl}}=Z_{\mathrm{cyl}}-Z_{\mathrm{cyl}}^{\mathrm{div}} & =\frac{i V_{26}}{2 \pi\left(8 \pi^{2} \alpha^{\prime}\right)^{13}} \int_{0}^{\infty} d s\left\{\eta(i s / \pi)^{-24}-\exp (2 s)-24\right\} \\
& =\frac{i V_{26}}{\left(8 \pi^{2} \alpha^{\prime}\right)^{13}} \int_{0}^{\infty} \frac{d t}{2 t}\left\{\frac{\eta(i t)^{-24}}{t^{13}}-\frac{\exp (2 \pi / t)}{t}-\frac{24}{t}\right\} .
\end{aligned}
$$

This expression, with the closed string divergences subtracted, is the one-loop correction to the tension according to the terminology introduced above. It is finite in the $t \rightarrow 0$ limit by construction but diverges in the $t \rightarrow \infty$ limit due to the behavior (2.6) of $\eta(i t)^{-24}$. This divergence is due to the open string tachyon. To make sense of this, consider as an example the one loop vacuum-to-vacuum amplitude for a point particle of mass $m$,

$$
Z\left(m^{2}\right)=i V_{26} \int_{0}^{\infty} \frac{d \ell}{2 \ell} \int \frac{d^{26} k}{(2 \pi)^{26}} e^{-\left(k^{2}+m^{2}\right) \ell / 2}=\frac{i V_{26}}{(2 \pi)^{13}} \int_{0}^{\infty} \frac{d \ell}{2 \ell} \frac{e^{-m^{2} \ell / 2}}{\ell^{13}} .
$$


Inserting the open string tachyon mass $\alpha^{\prime} m^{2}=-1$ and identifying $\ell=4 \pi \alpha^{\prime} t$, we find the same large $t$ divergence as in (2.9). More generally, the one loop Coleman-Weinberg effective potential is obtained from (2.10) by setting $m^{2}=V^{\prime \prime}(\phi)$ where $V(\phi)$ is the tree level potential; the effective potential diverges whenever the tree level potential satisfies $V^{\prime \prime}<0$. There exists a good understanding of this divergence, and a well defined prescription for rendering it finite, which we can apply in the string theory context.

To understand the issues involved, recall the physical meaning of the effective potential. Consider a scalar field theory with tree level potential $V(\phi)$. Let $\left\{\left|\Psi_{\bar{\phi}}\right\rangle\right\}$ denote the space of quantum states satisfying

$$
\left\langle\Psi_{\bar{\phi}}|\hat{\phi}(x)| \Psi_{\bar{\phi}}\right\rangle=\bar{\phi}=\text { constant }
$$

Now minimize the expectation value of the Hamiltonian in this space of states to obtain $E(\bar{\phi})$, assuming that such a minimum exists. Then, $V_{\text {eff }}(\phi)=E(\phi)$ is the effective potential. From this definition, it is straightforward to show that the effective potential is convex, $V_{\text {eff }}^{\prime \prime}(\phi) \geq 0$, which implies that $V(\phi)$ is a poor approximation to $V_{\text {eff }}(\phi)$ whenever $V^{\prime \prime}(\phi)<0$. For example, choose a $\bar{\phi}$ in a region between two adjacent minima of $V(\phi)$; then the state with minimum energy is not one concentrated near $\bar{\phi}$, but rather a linear combination of states localized near the minima.

It is now immediately clear that the effective potential for a tachyonic mass scalar field is ill-defined for any value of $\phi$, since the energy in the space of states with a fixed expectation value of $\hat{\phi}$ is unbounded from below. This explains the divergence in (2.9) and (2.10). It is also now clear that to obtain a finite result we should ask a more physically well defined question. A more reasonable question to ask is what is the energy of a state well localized near a given value of $\phi$. Note however that the answer to this question will depend on which particular state is chosen; furthermore, since no such state is an eigenstate of the Hamiltonian one can expect the energy to acquire an imaginary part:

$$
\left\langle\Psi\left|e^{-i H t}\right| \Psi\right\rangle=e^{-\operatorname{Im}(E) t-i \operatorname{Re}(E) t}
$$

for large $t$. It turns out that $\operatorname{Im}(E)$ is largely independent of the particular state chosen, and that for a particular "natural" choice of the state one can obtain both the real and imaginary parts by analytically continuing our original formula (2.10). This particular state corresponds to placing each Fourier mode $\phi_{k}$ in a minimum uncertainty wavepacket concentrated at the origin, except for the zero mode which is taken to be an eigenstate at the desired value of $\phi$. We refer to [29] for more details. 
To analytically continue (2.10), first divide the integration region into two parts (see [28] for a similar computation in a related context)

$$
I\left(m^{2}\right)=\int_{0}^{\infty} \frac{d \ell}{2 \ell} \frac{e^{-m^{2} \ell / 2}}{\ell^{13}}=\int_{0}^{\hat{\ell}} \frac{d \ell}{2 \ell} \frac{e^{-m^{2} \ell / 2}}{\ell^{13}}+\int_{\hat{\ell}}^{\infty} \frac{d \ell}{2 \ell} \frac{e^{-m^{2} \ell / 2}}{\ell^{13}} .
$$

The interesting divergence is in the second term, which we rewrite as

$$
\left(m^{2}\right)^{13} \int_{m^{2} \hat{\ell}}^{\infty} \frac{d x}{2 x} \frac{e^{-x / 2}}{x^{13}} .
$$

Now continue (2.14) to $m^{2}<0$ by taking an integration contour in the upper half plane that avoids the singularity at the origin. The analytic continuation yields an imaginary part for $m^{2}<0$,

$$
i \operatorname{Im}\left(I\left(m^{2}\right)\right)=-\frac{1}{2}\left(m^{2}\right)^{13} \oint_{C} \frac{d z}{2 z} \frac{e^{-z / 2}}{z^{13}}=-\frac{i \pi}{2 \cdot 13 !}\left(\frac{-m^{2}}{2}\right)^{13} .
$$

where $C$ is a contour circling the origin in the clockwise direction. Then from (2.10) this translates into an imaginary part for the energy given by

$$
\operatorname{Im}(E)=-\frac{V_{25}}{(2 \pi)^{13}} \operatorname{Im}\left(I\left(m^{2}\right)\right)=\frac{\pi V_{25}}{2 \cdot 13 !}\left(\frac{-m^{2}}{4 \pi}\right)^{13} .
$$

The decay rate of the system is given as usual by $\Gamma=2 \operatorname{Im}(E)$. The real part of $I\left(m^{2}\right)$ can also be extracted by evaluating the integral in the complex plane. It is easy to check that the result is independent of our choice for $\hat{\ell}$.

We can use precisely the same strategy to define our integral (2.9). Dividing up the range of integration, and using the asymptotic behavior (2.6), we can write (2.9) as

$$
\tilde{Z}_{\mathrm{cyl}}=\frac{i V_{26}}{\left(8 \pi^{2} \alpha^{\prime}\right)^{13}} \int_{\hat{t}}^{\infty} \frac{d t}{2 t} \frac{\exp (2 \pi t)}{t^{13}}+\tilde{Z}_{\mathrm{cyl}}^{\mathrm{finite}}
$$

$\tilde{Z}_{\text {cyl }}^{\text {finite }}$ is a well defined and purely imaginary expression given explicitly by

$$
\begin{aligned}
\tilde{Z}_{\mathrm{cyl}}^{\text {finite }}= & \frac{i V_{26}}{\left(8 \pi^{2} \alpha^{\prime}\right)^{13}} \int_{0}^{\hat{t}} \frac{d t}{2 t}\left\{\frac{\eta(i t)^{-24}}{t^{13}}-\frac{\exp (2 \pi / t)}{t}-\frac{24}{t}\right\} \\
& +\frac{i V_{26}}{\left(8 \pi^{2} \alpha^{\prime}\right)^{13}} \int_{\hat{t}}^{\infty} \frac{d t}{2 t}\left\{\frac{\eta(i t)^{-24}-\exp (2 \pi t)}{t^{13}}-\frac{\exp (2 \pi / t)}{t}-\frac{24}{t}\right\} .
\end{aligned}
$$

Next, we analytically continue in the open string tachyon mass exactly as before to obtain

$$
\tilde{Z}_{\mathrm{cyl}}=-\frac{i V_{26}}{\left(8 \pi^{2} \alpha^{\prime}\right)^{13}} \int_{C} \frac{d z}{2 z} \frac{\exp (-2 \pi z)}{z^{13}}+\tilde{Z}_{\mathrm{cyl}}^{\mathrm{finite}}
$$




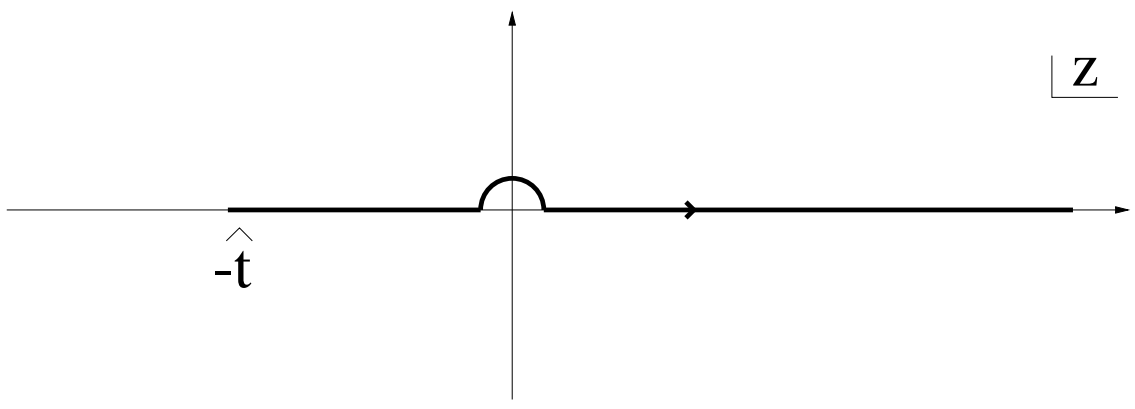

Fig. 1: Contour used in evaluating (2.19).

where $C$ is a contour running from $z=-\hat{t}$ to $z=\infty$ and avoiding the singularity in the origin; by convention we take $C$ to lie in the upper half complex plane, as shown in Fig. 1.

Therefore,

$$
\operatorname{Im}\left(i \tilde{Z}_{\mathrm{cyl}}\right)=\frac{\pi V_{26}}{2 \cdot 13 ! \cdot\left(4 \pi \alpha^{\prime}\right)^{13}}
$$

and

$$
\operatorname{Im}(E)=\frac{\pi V_{25}}{2 \cdot 13 ! \cdot\left(4 \pi \alpha^{\prime}\right)^{13}}
$$

The contribution to the real part of $E$ can also be extracted from (2.19).

Although we obtained $\operatorname{Im}(E)$ by a process of analytic continuation, we should again stress that it has a precise physical meaning in terms of the decay rate of an initial state localized at the unstable point of the tachyon potential. In the string theory context (2.21) gives the leading order decay rate of a bosonic D25-brane. We also note that although we found it convenient to compute the decay rate in terms of the imaginary part of a loop amplitude, it is essentially a tree level quantity in the sense that it could have been obtained by solving the tree level equations of motion.

Let us now say a few words about the manner in which the initial state decays. One's initial intuition is that the D25-brane decays via the tachyon rolling homogeneously down the potential. However, the actual decay process is inhomogeneous and proceeds by the amplification of long wavelength modes of the tachyon (spinodal decomposition). In infinite volume the zero mode of the tachyon is a classical variable and can be taken to be fixed at the top of the potential. Modes of the tachyon obeying $\vec{k}^{2}+m^{2}<0$ are unstable, and grow exponentially in time, with the longest wavelengths growing most rapidly. Thus there will be large regions of space in which the tachyon rolls down the left hand side of the potential, and large regions in which it rolls down the right hand side. In the bosonic string the potential seems to be unbounded from below in one direction, and so the actual 
outcome of the decay process is uncertain. For the superstring the potential is bounded from below, and the decay will eventually terminate in a distribution of topological defects.

\section{Classical and Quantum BSFT}

We would like to generalize the computations in the previous section to allow off-shell open strings, such as a tachyon condensate. In this section we review BSFT and attempt to define loop corrections in BSFT by quantizing the tree level action as an ordinary field theory.

\subsection{Classical BSFT}

Boundary string field theory (BSFT) describes the off-shell dynamics of open strings in a fixed on-shell background of closed strings. An open string field configuration corresponds to a boundary term in the world-sheet action of the string. Specifying a boundary term means giving the background values of the various modes of the open string. In the case of bosonic open string theory, to which we specialize now, these modes are tachyons, gauge potentials and massive string states. BSFT formally defines an action functional on this space of string field configurations, equal to the renormalized partition function on the unit disk of the world-sheet theory, up to a correction involving the world-sheet beta functions.

In formulas, one starts with a world-sheet theory

$$
\mathcal{S}=\mathcal{S}_{0}+\int_{0}^{2 \pi} \frac{d \tau}{2 \pi} \mathcal{V}
$$

where $\mathcal{S}_{0}$ is conformally invariant and the boundary perturbation $\mathcal{V}$ can be expanded as

$$
\mathcal{V}=\sum_{i} \lambda^{i} \mathcal{V}_{i}
$$

The world-sheet couplings correspond to the modes of spacetime fields. In terms of the $\beta$-functions for these world-sheet couplings, the classical space-time action $S(\lambda)$ is defined as [22,24]

$$
S=\left(\beta^{i} \frac{\partial}{\partial \lambda^{i}}+1\right) Z(\lambda)
$$

where $Z$ is the renormalized disk partition function of the theory defined by (3.1). In

fact, the $\beta$-function term in (3.3) subtracts the divergence due to the infinite volume of the Möbius group, and could be viewed as part of the renormalization procedure (it 
corresponds to a singular wave function renormalization of the tachyon). It also ensures that $S$ has extrema when the boundary theory is conformal 1 . Note that for a conformally invariant boundary action $\left(\beta^{i}=0\right)$ the spacetime action equals the disk partition function.

For later reference we note that another way of writing (3.3) is [24, 34]

$$
\frac{\partial S}{\partial \lambda^{i}}=\beta^{j} G_{i j}(\lambda)
$$

where $G_{i j}$ is the metric on the space of theories (we shall not need its explicit form in this paper).

The fact that the renormalized disk partition function can be identified with the spacetime action is familiar from the sigma model approach to string theory [39] (for reviews see e.g. 40,41,42]). In that approach, most attention has gone to on-shell, renormalizable boundary interactions, and usually one constructs the action in a derivative expansion. It is convenient to work in Euclidean spacetime signature, because then the world-sheet theory is unitary. Almost by construction, the bare (unrenormalized) disk partition function is the generating functional of S-matrix elements (up to a factor of the Möbius volume that has to be divided out). Divergences arise due to the exchange of zero momentum massless open string modes and of tachyonic modes with low enough momentum, as is clear from the Schwinger parameterization of propagators,

$$
\left(p^{2}+m^{2}\right)^{-1}=\int_{0}^{\infty} d s e^{-\left(p^{2}+m^{2}\right) s} .
$$

From the world-sheet point of view these are short distance divergences and are removed by renormalizing the partition function. Thus, at least in Euclidean signature, the renormalized partition function generates diagrams that are one-particle irreducible (1PI) with respect to those massless and tachyonic lines. The terms containing only these renormalizable modes correspond to their effective action with the unrenormalizable modes integrated out. It has been checked in various examples that, for massless external open string states and in a derivative expansion, the S-matrix of perturbative string theory can be reproduced from this effective action [40,43,41]. One just computes the Feynman diagrams corresponding to the exchanges of the massless and tachyonic modes that were subtracted out. Some checks have also been performed for tachyonic external states [44].

1 In the case of the superstring the BSFT action simply equals the renormalized disk partition function without $\beta$-function corrections [34, 35, 36, 37, 38]. 
BSFT is an extension of the sigma model approach which is non-trivial in several ways. First, one wants to be able to discuss off-shell open strings (the closed string background remains on-shell). Further, one would like to describe all open string modes, including the ones corresponding to irrelevant (unrenormalizable) boundary interactions. And finally, one would like to go beyond the low energy expansion. These complications are challenging, and at present it is unclear whether the ambitions of BSFT can be realized even at the classical level. In particular it seems to require additional information to treat the irrelevant interactions exactly but it is possible to incorporate them perturbatively by expanding in these operators.

As discussed above, a conventional renormalization prescription in Euclidean signature produces a sigma-model action with all irrelevant open string modes integrated out classically. This action is inappropriate away from the classical and low energy limits. Instead, what one wants is an action that generates 1PI diagrams with respect to all modes, whether massless, tachyonic, or massive. This would be the sort of action that deserves to be called a string field theory; for instance, the much studied cubic bosonic open string field theory [45] is of this nature (see [46, 47,48,49] and references therein for some recent developments). Given such an action, off-shell amplitudes for arbitrary external states are computed from Feynman diagrams as in an ordinary field theory. It may also be necessary to add new vertices at each order in perturbation theory in order to recover the correct on-shell loop amplitudes.

\subsection{Quantum BSFT}

Let us summarize the situation: tree level BSFT leads to a spacetime "action" with the irrelevant modes integrated out classically. Unfortunately such an action is not an acceptable starting point for including loop corrections; for instance, the effects of massive states running in loops cannot be taken into account if they have been integrated out classically.

At this point one might simply give up on defining loop amplitudes starting from tree-level BSFT, concluding that BSFT necessarily is an effective field theory description limited to the light open string modes. This pessimistic view is consistent with the fact that the successes of BSFT so far only probe relevant and marginal operators at the classical level. In the remainder of this section we discuss the alternative and more optimistic view that a more fundamental classical action can be defined which describes all open string modes and generates truly 1PI diagrams. 
What we need is a different renormalization prescription that subtracts, from the spacetime point of view, divergences due to the exchange of any on-shell open string state. Such a prescription would seem to arise most naturally in Lorentzian signature where the regularized propagator takes the form

$$
\frac{1}{p^{2}-m^{2}}=-\int_{0}^{\ln \Lambda} d s e^{\left(p^{2}-m^{2}\right) s} .
$$

Subtracting the logarithmic divergences will then remove the exchange of any on-shell state. This prescription is in fact not entirely well-defined as it stands because now the underlying CFT is non-unitary; however, it serves to indicate what is needed. In the following discussion we will only rely on a few structural features of the action. In particular, we will not need a precise definition of our renormalization prescription beyond saying that it should subtract divergences from all on-shell exchanges.

According to (3.4), the equations of motion are $\beta_{i}=0$ where $i$ enumerates a complete set of operators. These are not the familiar sigma-model conditions for conformal invariance: to recover such standard results as the Born-Infeld action, one would need to eliminate the massive fields by solving their equation of motion. Banks and Martinec have defined a string field theory with these "complete" equations of motion [50] and it was shown that they imply the correct tree level S-matrix amplitudes [51]. Their $\beta_{i}$ actually refer to the Wilsonian beta functions but the relation between Wilsonian and "conventional" beta functions corresponds to a redefinition of couplings so one expects the same success in our construction.

We can make the action slightly more explicit by expanding it around the classical solution $\lambda_{i}=0$

$$
S=\sum_{n=2}^{\infty} S_{i_{1} \cdots i_{n}} \lambda^{i_{1}} \cdots \lambda^{i_{n}} .
$$

For constant tachyon background $\int \frac{d \tau}{2 \pi} T=T$ we have $Z\left(T, \lambda^{i}\right)=e^{-T} Z\left(0, \lambda^{i}\right)$ so, after taking (3.3) into account, the expansion (3.7) takes the form

$$
S=\sum_{n=2}^{\infty}\left(a_{i_{1} \cdots i_{n}}+b_{i_{1} \cdots i_{n}} T\right) e^{-T} \lambda^{i_{1}} \cdots \lambda^{i_{n}}
$$

where now the $\lambda^{i}$ exclude $T$.

We can try to compute loop amplitudes using the action (3.8). By construction, the loop amplitudes obtained this way will factorize correctly in the open string channel, and it seems plausible that on-shell we will recover the correct S-matrix amplitudes 2. However,

2 It would of course be worthwhile to check this explicitly as was done in the cubic theory [52]. 
the open string loop amplitudes should also factorize properly in the closed string channel

and it is far from obvious how this is supposed to come about 3 Moreover, the theory described by (3.8) apparently becomes strongly coupled at $T \rightarrow \infty$, which is the location of the closed string vacuum. This is puzzling, because in the next section we will define loop amplitudes that automatically factorize correctly in the closed string channel but are weakly coupled at the closed string vacuum. It will be an interesting question to find the precise relation between these two descriptions.

\section{Loop Corrections to the Tachyon Action}

The purpose of this section is to propose a prescription for loop computations in BSFT that circumvents the problems discussed in section 3. We first explain the problems with a naïve annulus prescription. Then the BSFT couplings between open and closed strings are discussed. Finally, we combine the ingredients and find a factorization condition that defines loop amplitudes.

\subsection{The Partition Function on the Annulus?}

The classical BSFT action arises from evaluating the disk partition function with the boundary interaction

$$
S_{\mathrm{bndy}}=\int \frac{d \tau}{2 \pi} T(X(\tau))
$$

To define a loop correction one might propose simply adding an additional boundary to the disk (weighted by $e^{-S_{\text {bndy }}}$ ) and then integrating over moduli. This of course would be in direct analogy to how one computes loop corrections to on-shell quantities.

In the on-shell case one has a freedom in the choice of a world-sheet Weyl factor, and it is customary to use this freedom to let the world-sheet have the geometry of either a cylinder or an annulus. Conformal invariance guarantees that on-shell amplitudes are independent of this choice. But once we break conformal invariance these world-sheets no longer give equivalent results, and one is left with an ambiguity as to the choice of Weyl factor. This ambiguity did not arise at the level of the disk because the definition of BSFT demands that the Weyl factor be rotationally invariant, and the remaining freedom can be compensated for by a redefinition of couplings. One possible approach is to contemplate

3 See [53] for an alternative string field theory that makes factorization manifest, and which bears resemblance to our approach in section 4. 
integrating over Weyl factors, but this seems challenging both technically and conceptually, and also seems against the spirit of tree level BSFT which assumes a fixed Weyl factor.

To gain a more physical understanding of the issues involved, let us see what goes wrong when we simply decree that the annulus is the preferred world-sheet. We take our annulus to have a unit outer radius and an inner radius a. For Neumann boundary conditions (no boundary interaction) the annulus amplitude is

$$
Z_{\mathrm{ann}}=\int_{0}^{1} \frac{d a}{a^{3}} \prod_{n=1}^{\infty}\left(1-a^{2 n}\right)^{-24}
$$

Now include a constant tachyon $T$. This simply introduces a factor

$$
e^{-S_{\text {bndy }}}=e^{-(1+a) T}
$$

so the annulus amplitude becomes

$$
Z_{\mathrm{ann}}(T)=\int_{0}^{1} \frac{d a}{a^{3}} \prod_{n=1}^{\infty}\left(1-a^{2 n}\right)^{-24} e^{-(1+a) T} .
$$

The annulus amplitude diverges in the small $a$ region due to the exchange of tachyonic and massless closed string modes,

$$
Z_{\mathrm{ann}}(T)=\int_{0}^{1} \frac{d a}{a^{3}} e^{-T}\left(1-a T+\left(24+\frac{1}{2} T^{2}\right) a^{2}+O\left(a^{3}\right)\right) .
$$

In particular, cutting off the lower limit of integration we find a logarithmic divergence equal to

$$
-\left(24+\frac{1}{2} T^{2}\right) e^{-T} \log a_{\min } .
$$

In the discussion of the on-shell loop-amplitude in section 2 we encountered this divergence at $T=0$ and removed it using the Fischler-Susskind mechanism; i.e. it was attributed to the tree level exchange of dilatons and gravitons. The amplitude for graviton exchange is of the form

$$
\left.\frac{T^{\mu \nu} T_{\mu \nu}}{k^{2}}\right|_{k^{2}=0} \rightarrow T^{\mu \nu} T_{\mu \nu} \log a_{\min }
$$

and from tree level BSFT we know that the energy momentum tensor in the presence of a constant tachyon is proportional to the tachyon potential, $T_{\mu \nu} \sim(1+T) e^{-T} \eta_{\mu \nu}$. The dilaton contribution is of similar form. So we find a discrepancy with (4.6) : the annulus divergence cannot be interpreted as due to exchange of gravitons that couple to the energy 
momentum tensor of the open strings. It seems unlikely that one could develop a consistent formalism in which gravity does not couple to the energy momentum tensor, and so we conclude that annular world-sheets leads to inconsistencies.

The most obvious problem with the annulus is that it treats the two boundaries asymmetrically, while closed string factorization implies that the two boundaries should be on an equal footing. This immediately suggests that cylindrical world-sheets are better candidates. In fact, we will argue that the cylinder also fails to factorize correctly, but that the discrepancies only arise for nonconstant tachyon backgrounds.

Rather than making an ad hoc choice of world-sheet and then checking for factorization, we will take factorization as our starting point and derive loop amplitudes based on this physical condition. This is a powerful principle because an amplitude with any number of boundaries (but no handles) can be represented as a tree level closed string process. We will concentrate on the case of two boundaries, which requires us to know the closed string propagator as well as the vertex coupling open strings to a single closed string.

\subsection{Tree Level Couplings to Closed Strings}

We first determine the vertices by evaluating the disk amplitude with insertion of a closed string vertex operator. One is accustomed to using conformal invariance to freely choose the position of the closed string vertex operator, or to use an integrated vertex operator. But in the presence of a non-conformally invariant boundary interaction, or for an off-shell closed string vertex operator, these choices are inequivalent. The correct procedure is to use integrated vertex operators. (This certainly seems like the most symmetrical choice and arises from a sum over surfaces approach).

To show this, consider the disk partition function in the absence of a closed string vertex operator,

$$
Z=\int D X e^{-S_{\mathrm{bulk}}-S_{\mathrm{bndy}}}
$$

with

$$
S_{\mathrm{bulk}}=\frac{1}{4 \pi \alpha^{\prime}} \int d^{2} x \sqrt{\gamma} \gamma^{a b} G_{\mu \nu} \partial_{a} X^{\mu} \partial_{b} X^{\nu}
$$

where we take $G_{\mu \nu}=\eta_{\mu \nu}$. Taking a general tachyon boundary interaction $T(X)$, we will find

$$
Z[T(X)]=Z\left[T, \eta^{\mu \nu} \partial_{\mu} T \partial_{\nu} T, \ldots\right]
$$


Now consider the vertex operator corresponding to a zero momentum graviton, $V \sim$ $h_{\mu \nu} \partial X^{\mu} \bar{\partial} X^{\nu}$. Such a zero momentum graviton is of course just a coordinate transformation, and the effect on the disk amplitude (4.10) should just be to replace $\eta_{\mu \nu} \rightarrow \eta_{\mu \nu}+h_{\mu \nu}$. This will be satisfied provided that the graviton vertex operator is defined as the functional derivative of the bulk action with respect to the metric; i.e.

$$
V=\frac{\delta S_{\mathrm{bulk}}}{\delta G_{\mu \nu}} h_{\mu \nu} \sim \int d^{2} x \sqrt{\gamma} \gamma^{a b} h_{\mu \nu} \partial_{a} X^{\mu} \partial_{b} X^{\nu}
$$

So general covariance demands that we choose an integrated vertex operator for the graviton. Choosing instead, say, a vertex operator fixed at the origin, one will find that the graviton couples not to the standard energy momentum tensor but to something else, and this will in all likelihood lead to an inconsistency. Similarly, we expect that the full set of closed string gauge invariances require one to use integrated vertex operators in general, and we will assume this from now on.

So our vertex coupling a single closed string state with momentum $p$ to any number of open string is given by evaluating

$$
Z_{\text {disk }}\left(V_{I}(p) ; S_{\text {bndy }}\right)=\int D X e^{-S_{\text {bulk }}-S_{\text {bndy }}} V_{I}(p)
$$

where $V_{I}(p)$ is an integrated vertex operator for the closed string state $I$. In evaluating (4.12) one will encounter divergences when the vertex operator approaches the boundary of the disk. Furthermore, to obtain the proper normalization of (4.12) one should divide by the volume of the Möbius group, but this is also divergent. It was shown in [54] how to renormalize these divergences. In the on-shell case the result is the same as if one had fixed the vertex operator at the origin and divided by the (finite) volume of the unbroken subgroup of the Möbius group.

\subsection{Loops via Factorization}

To construct a "cylinder amplitude" using the above vertices we first factorize the standard cylinder amplitude (with a conformally invariant boundary interaction) in terms of disk amplitudes with closed string vertex operators fixed at the origin:

$$
Z_{\text {cyl }}\left(S_{\text {bndy }}\right)=\sum_{I} \int \frac{d^{26} p}{(2 \pi)^{26}} Z_{\text {disk }}\left(V_{I}^{\text {fixed }}(p) ; S_{\text {bndy }}\right) \frac{1}{p^{2}+m_{I}^{2}} Z_{\text {disk }}\left(V_{I}^{\text {fixed }}(-p) ; S_{\text {bndy }}\right),
$$


where $I$ enumerates all closed string states. One way of deriving this formula is in the boundary state formalism, see [55] for a review. If we simply defined off-shell amplitudes by allowing nonconformal $S_{\text {bndy }}$ in (4.13) then factorization would imply that the couplings to closed strings were given by the disk amplitude with fixed vertex operators inserted; but those couplings would be incorrect since we showed above that one must use integrated vertex operators.

To correct for this we need to relate the disk amplitudes with fixed and integrated vertex operators. Our integrated vertex operators are integrals over the disk of conformal tensors of dimension $(\Delta, \Delta)$ with $\Delta=1+\alpha^{\prime}\left(p^{2}+m_{I}^{2}\right) / 4$. We can perform an $S L(2, R)$ transformation to bring any point in the interior of the disk to the origin. If the boundary interaction is conformally invariant we can use this to transform a disk amplitude with an integrated vertex operator into one with a fixed vertex operator, picking up in the process a function of $\Delta-1$; so for conformally invariant boundary interactions

$$
Z_{\mathrm{disk}}\left(V_{I}^{\mathrm{fixed}}(p) ; S_{\mathrm{bndy}}\right)=f\left(p^{2}+m_{I}^{2}\right) Z_{\mathrm{disk}}\left(V_{I}(p) ; S_{\mathrm{bndy}}\right)
$$

Since fixed and integrated vertex operators are equivalent onshell we have $f(0)=1$. Using (4.14) in 4.13) we find

$$
Z_{\mathrm{cyl}}\left(S_{\mathrm{bndy}}\right)=\sum_{I} \int \frac{d^{26} p}{(2 \pi)^{26}} Z_{\mathrm{disk}}\left(V_{I}(p) ; S_{\mathrm{bndy}}\right) \frac{f\left(p^{2}+m_{I}^{2}\right)^{2}}{p^{2}+m_{I}^{2}} Z_{\mathrm{disk}}\left(V_{I}(-p) ; S_{\mathrm{bndy}}\right)
$$

We define general off-shell loop-amplitudes by allowing arbitrary $S_{\mathrm{bndy}}$ in this formula. This definition has all the properties we desire: by construction it reproduces the correct on-shell amplitudes and also factorizes correctly in the closed string channel. In particular, the amplitude for graviton exchange is given by the correct energy momentum tensor of the open strings. We stress again that our result is not the same as simply evaluating the cylinder diagram with arbitrary boundary interactions.

In general it will be quite involved to actually evaluate (4.15). However, it is trivial for a constant tachyon background because then $Z_{\text {disk }}(T)=e^{-T} Z_{\text {disk }}(0)$ and so the sum over $I$ in (4.15) can be evaluated using the on-shell result (4.13). It is appropriate to use the disk amplitude with the subtraction of the infinite Möbius volume, i.e. the tree level action (3.3). This disk amplitude is just the tree level tachyon potential. We therefore find that the tachyon potential is loop corrected by

$$
V_{\text {loop }}=(V(T))^{2} \tilde{Z}_{\text {cyl }} .
$$


where $\tilde{Z}_{\text {cyl }}$ was defined in (2.19) and the tree level potential is $V(T)=(1+T) e^{-T}$.

We can alternatively write our result (4.15) as

$$
Z_{\text {cyl }}\left(S_{\text {bndy }}\right)=\int D X D Y \Psi(X) G(X, Y) \Psi(Y)
$$

where $\Psi(X)=e^{-S_{\text {bndy }}(X)}$ represent the boundary state. This defines $G(X, Y)$ as an offshell closed string propagator. It would be interesting to compare with the version of the propagator studied in [56].

\section{Comments}

In summary, we have discussed two proposals for the off-shell loop amplitude: the "open string" definition of section 3 (if realizable) quantizes the tree level BSFT action directly and the "closed string" definition of section 4 demands factorization in the closed string channel. We conclude by comparing these definitions:

(1) The two approaches plausibly agree for on-shell external open string states. Our closed string approach agrees with standard on-shell amplitudes by construction. Demonstrating this in the open string approach requires more analysis, but we see no reason in principle why this will not occur.

(2) They have different factorization properties. By construction the open string approach will factorize correctly in the open string channel, and similarly for the closed string approach, but correct factorization in the opposite channel may not work off-shell. A similar situation pertains to cubic string field theory, where it was shown that off-shell open string amplitudes have unphysical poles in the closed string channel [52]. It is not hard to understand where problems can arise. Closed string poles are high energy effects in open string field theory but when one goes off-shell and breaks conformal invariance on the world-sheet, renormalization of divergences can disturb this high energy behavior.

(3) They generate different amplitudes off-shell. Consider amplitudes in the background of a constant tachyon. The open string action (3.8) gives propagators weighted by $e^{T}$ and vertices weighted by $e^{-T}$ (times a possible linear term); so the loop expansion parameter in the open string approach is proportional to $e^{T}$ and a string diagram with $n$ boundaries picks up a factor $\left(e^{T}\right)^{n-1}$. On the other hand, in the closed string approach the tachyon enters as $e^{-T}$ in each of the "constituent" disk amplitudes 
and the closed string propagators are $T$ independent; therefore a diagram with $n$ boundaries is proportional to $\left(e^{-T}\right)^{n}$. These differences are most dramatic for large values of the tachyon, i.e. near the closed string vacuum. Here the open string description is strongly coupled while the closed string description is weakly coupled.

(4) Usually two prescriptions which agree on-shell but differ off-shell are related by a field redefinition. It is therefore tempting to speculate that an equivalence exists between the strongly coupled open string description and the weakly coupled closed string description; in other words, there is a novel kind of duality. It would hardly be surprising that closed strings are the appropriate variables around the closed string vacuum. This type of relation would also be reminiscent of the AdS/CFT correspondence [57,58,59. In any event, one lesson from AdS/CFT is that off-shell open strings can be dual to on-shell closed strings, and as such be observable. This may serve as further motivation for our insisting on factorization of the "cylinder" amplitude even with non-conformally invariant boundary interactions.

Note added: As this manuscript was being prepared, the preprint [60] appeared which contains the computation of sec 2.3 .

Acknowledgements: We thank R. Gopakumar, E. Martinec, L. Rastelli, S. Shatashvili, A. Tseytlin, E. Witten, B. Zwiebach and particularly D. Kutasov for discussions. This work was supported in part by NSF grant PHY-9901194 and by DOE grant DE-FG0290ER40560. FL was supported in part by a Robert R. McCormick fellowship. FL thanks Rutgers University for hospitality during parts of this work. 


\section{References}

[1] V. A. Kostelecky and S. Samuel, "On A Nonperturbative Vacuum For The Open Bosonic String", Nucl. Phys. B 336, 263 (1990).

[2] A. Sen, "Non-BPS states and branes in string theory," hep-th/9904207.

[3] A. Sen and B. Zwiebach, "Tachyon condensation in string field theory," JHEP 0003, 002 (2000) hep-th/9912249.

[4] A. Strominger, "Closed Strings In Open String Field Theory," Phys. Rev. Lett. 58, 629 (1987).

[5] M. Srednicki and R. P. Woodard, "Closed From Open Strings In Witten's Theory," Nucl. Phys. B 293, 612 (1987).

[6] J. A. Harvey, P. Kraus, F. Larsen and E. J. Martinec, "D-branes and strings as noncommutative solitons," JHEP 0007, 042 (2000) [hep-th/0005031].

[7] G. Gibbons, K. Hori and P. Yi, "String fluid from unstable D-branes," Nucl. Phys. B 596, 136 (2001) hep-th/0009061.

[8] A. Sen, "Fundamental strings in open string theory at the tachyonic vacuum", hepth/0010240].

[9] A. A. Gerasimov and S. L. Shatashvili, "Stringy Higgs mechanism and the fate of open strings", JHEP 0101, 019 (2001) hep-th/0011009.

[10] G. Chalmers, "Open string decoupling and tachyon condensation", hep-th/0103056.

[11] A. Sen, "Universality of the tachyon potential," JHEP 9912, 027 (1999) hepth/9911116.

[12] P. Yi, "Membranes from five-branes and fundamental strings from Dp branes," Nucl. Phys. B 550, 214 (1999) [hep-th/9901159].

[13] A. Sen, "Supersymmetric world-volume action for non-BPS D-branes," JHEP 9910, 008 (1999) hep-th/9909062.

[14] O. Bergman, K. Hori and P. Yi, "Confinement on the brane," Nucl. Phys. B 580, 289 (2000) hep-th/0002223.

[15] M. Kleban, A. E. Lawrence and S. Shenker, "Closed strings from nothing", hepth/0012081.

[16] J. A. Harvey, D. Kutasov and E. J. Martinec, "On the relevance of tachyons", hepth/0003101].

[17] D. Kutasov, M. Marino and G. Moore, "Some exact results on tachyon condensation in string field theory", JHEP 0010, 045 (2000) hep-th/0009148.

[18] J. D. Blum and K. R. Dienes, "Strong/weak coupling duality relations for nonsupersymmetric string theories", Nucl. Phys. B 516, 83 (1998) [hep-th/9707160].

[19] O. Bergman and M. R. Gaberdiel, "Dualities of type 0 strings", JHEP 9907, 022 (1999) hep-th/9906055. 
[20] M. S. Costa and M. Gutperle, "The Kaluza-Klein Melvin solution in M-theory", JHEP 0103, 027 (2001) hep-th/0012072.

[21] E. Witten, "On background independent open string field theory", Phys. Rev. D 46, 5467 (1992) [hep-th/9208027.

[22] E. Witten, "Some computations in background independent off-shell string theory", Phys. Rev. D 47, 3405 (1993) hep-th/9210065.

[23] S. L. Shatashvili, "Comment on the background independent open string theory", Phys. Lett. B 311, 83 (1993) hep-th/9303143.

[24] S. L. Shatashvili, "On the problems with background independence in string theory", hep-th/9311177.

[25] A. A. Gerasimov and S. L. Shatashvili, "On exact tachyon potential in open string field theory", JHEP 0010, 034 (2000) hep-th/0009103.

[26] W. Fischler and L. Susskind, "Dilaton Tadpoles, String Condensates And Scale Invariance. 2", Phys. Lett. B 173, 262 (1986).

[27] C. G. Callan, C. Lovelace, C. R. Nappi and S. A. Yost, "String Loop Corrections To Beta Functions," Nucl. Phys. B 288, 525 (1987).

[28] N. Marcus, "Unitarity And Regularized Divergences In String Amplitudes", Phys. Lett. B 219, 265 (1989).

[29] E. J. Weinberg and A. Wu, "Understanding Complex Perturbative Effective Potentials", Phys. Rev. D 36, 2474 (1987).

[30] A. H. Guth and S. Pi, "The Quantum Mechanics Of The Scalar Field In The New Inflationary Universe", Phys. Rev. D 32, 1899 (1985).

[31] K. S. Viswanathan and Y. Yang, "Tachyon condensation and background independent superstring field theory", hep-th/0104099.

[32] M. Alishahiha, "One-loop correction of the tachyon action in boundary superstring field theory", hep-th/0104164.

[33] N. D. Lambert and I. Sachs, "String loop corrections to stable non-BPS branes", JHEP 0102, 018 (2001) hep-th/0010045].

[34] D. Kutasov, M. Marino and G. Moore, "Remarks on tachyon condensation in superstring field theory", hep-th/0010108.

[35] P. Kraus and F. Larsen, "Boundary string field theory of the D D-bar system", Phys. Rev. D 63, 106004 (2001) hep-th/0012198.

[36] T. Takayanagi, S. Terashima and T. Uesugi, "Brane-antibrane action from boundary string field theory", JHEP 0103, 019 (2001) [hep-th/0012210.

[37] M. Marino, "On the BV formulation of boundary superstring field theory", hepth/0103089.

[38] V. Niarchos and N. Prezas, "Boundary superstring field theory", hep-th/0103102.

[39] E. S. Fradkin and A. A. Tseytlin, "Effective Field Theory From Quantized Strings", Phys. Lett. B 158, 316 (1985). 
[40] A. A. Tseytlin, "Sigma Model Approach To String Theory", Int. J. Mod. Phys. A 4, 1257 (1989).

[41] A. A. Tseytlin, "Sigma model approach to string theory effective actions with tachyons", hep-th/0011033.

[42] A. A. Tseytlin, "Renormalization Group And String Loops", Int. J. Mod. Phys. A 5, 589 (1990).

[43] O. D. Andreev and A. A. Tseytlin, "Partition Function Representation For The Open Superstring Effective Action: Cancellation Of Mobius Infinities And Derivative Corrections To Born-Infeld Lagrangian", Nucl. Phys. B 311, 205 (1988).

[44] S. A. Frolov, "On off-shell structure of open string sigma model", hep-th/0104042.

[45] E. Witten, "Noncommutative Geometry And String Field Theory", Nucl. Phys. B 268, 253 (1986).

[46] I. Ellwood and W. Taylor, "Open string field theory without open strings", hepth/0103085.

[47] L. Rastelli, A. Sen and B. Zwiebach, "Boundary CFT Construction of D-branes in Vacuum String Field Theory", hep-th/0105168.

[48] D. J. Gross and W. Taylor, "Split string field theory. I", hep-th/0105059].

[49] T. Kawano and K. Okuyama, "Open string fields as matrices", hep-th/0105129.

[50] T. Banks and E. Martinec, "The Renormalization Group And String Field Theory", Nucl. Phys. B 294, 733 (1987).

[51] J. Hughes, J. Liu and J. Polchinski, "Virasoro-Shapiro From Wilson," Nucl. Phys. B 316, 15 (1989).

[52] D. Z. Freedman, S. B. Giddings, J. A. Shapiro and C. B. Thorn, "The Nonplanar One Loop Amplitude In Witten's String Field Theory", Nucl. Phys. B 298, 253 (1988).

[53] B. Zwiebach, "Quantum open string theory with manifest closed string factorization", Phys. Lett. B 256, 22 (1991).

[54] J. Liu and J. Polchinski, "Renormalization Of The Mobius Volume", Phys. Lett. B 203, 39 (1988).

[55] P. Di Vecchia and A. Liccardo, "D branes in string theory. I", hep-th/9912161.

[56] A. Cohen, G. Moore, P. Nelson and J. Polchinski, "An Off-Shell Propagator For String Theory", Nucl. Phys. B 267, 143 (1986).

[57] J. Maldacena, "The large N limit of superconformal field theories and supergravity," Adv. Theor. Math. Phys. 2, 231 (1998) [Int. J. Theor. Phys. 38, 1113 (1998)] hepth/9711200].

[58] S. S. Gubser, I. R. Klebanov and A. M. Polyakov, "Gauge theory correlators from non-critical string theory," Phys. Lett. B 428, 105 (1998) hep-th/9802109.

[59] E. Witten, "Anti-de Sitter space and holography", Adv. Theor. Math. Phys. 2, 253 (1998) hep-th/9802150].

[60] K. Bardakci and A. Konechny, "Tachyon condensation in boundary string field theory at one loop", hep-th/0105098. 